\title{
Simultaneous Ingestion of Fructose and Fat Exacerbates Postprandial Exogenous Lipidemia in Young Healthy Japanese Women
}

\author{
Hiromi Saito ${ }^{1}$, Mieko Kagaya ${ }^{2}$, Maiko Suzuki ${ }^{1}$, Akihiro Yoshida $^{3}$ and Michitaka Naito ${ }^{1}$ \\ ${ }^{1}$ Division of Nutrition \& Health, School \& Graduate School of Life Studies, Sugiyama Jogakuen University, Nagoya, Japan \\ ${ }^{2}$ Division of Cookery Science, School of Life Studies, Sugiyama Jogakuen University, Nagoya, Japan \\ ${ }^{3}$ Department of Clinical Laboratory, Nakatsugawa Municipal General Hospital, Nakatsugawa, Japan
}

\begin{abstract}
Aim: To investigate the acute effects of the simultaneous ingestion of fructose and fat on postprandial lipoprotein metabolism in healthy young women.

Methods: Nine young healthy Japanese women with a normal weight (body mass index: $18.5 \leq-<25$ $\mathrm{kg} / \mathrm{m}^{2}$ ), a normal ovarian cycle and an apolipoprotein E 3/3 phenotype were enrolled as participants and studied on four occasions. At each session, the subjects ingested one of four beverages containing either glucose or fructose $(0.5 \mathrm{~g} / \mathrm{kg}$ body weight each) with or without OFTT cream $(1 \mathrm{~g} / \mathrm{kg}, 0.35 \mathrm{~g} /$ $\mathrm{kg}$ as fat) in a randomized crossover design. Blood samples were collected at baseline and at $0.5,1,2$, 4 and 6 hours after ingestion.

Results: The ingestion of fructose combined with fat led to significantly higher rises in the serum triglyceride (TG), remnant-like particle (RLP)-TG, remnant lipoprotein-cholesterol (RemL-C) and apolipoprotein B-48 (apoB48) concentrations with delayed peaks compared with that observed following ingestion of the other three types of beverages. The incremental area under the curve $(\triangle$ AUC)-TG and $\triangle A U C$-apoB 48 were larger than those observed for the ingestion of fat only. The serum RLP-TG and apoB48 concentrations returned to the fasting levels ( 0 hours) at the end of the test ( 6 hours) following the ingestion of fat only; however, these concentrations did not return to the fasting levels following the intake of fructose combined with fat.

Conclusions: These findings suggest a delay in the clearance of intestinal TG-rich lipoproteins, namely chylomicron and its remnant, following the ingestion of fructose combined with fat. The simultaneous ingestion of fructose and fat markedly enhances postprandial exogenous lipidemia in young healthy Japanese women.
\end{abstract}

J Atheroscler Thromb, 2013; 20:591-600.

Key words: Fructose, Postprandial lipidemia, Triglyceride, Apolipoprotein B-48, Remnant

\section{Introduction}

High fructose corn syrup (HFCS) was developed industrially as an alternative to sucrose in the USA over the period from 1970 to $1985^{11}$. In Japan, the consumption of HFCS has been increasing since

Address for correspondence: Michitaka Naito, Division of Nutrition \& Health, School \& Graduate School of Life Studies, Sugiyama Jogakuen University, 17-3, Hoshigaokamotomachi, Chikusa-ku, Nagoya 464-8662, Japan

E-mail: naito@sugiyama-u.ac.jp

Received: November 27, 2012

Accepted for publication: February 26, 2013
1980, while that of sucrose has decreased ${ }^{2)}$, and HFCS currently accounts for approximately $40 \%$ of the total consumption of sugar, ranking just behind the USA ${ }^{3)}$. The products in which HFCS is used include soft drinks, which account for approximately $50 \%$ of the total consumption of $\mathrm{HFCS}^{2)}$. However, detailed research has not yet been conducted on the daily consumption of HFCS and fructose by Japanese people.

In the USA, many studies have shown a relationship between the intake of sugar-sweetened beverages and weight gain ${ }^{4}$, and the exaggerated postprandial triglyceride (TG) response observed following the ingestion of HFCS has been shown to be caused by 
the fructose component ${ }^{5)}$. Some studies have shown that the plasma concentration of TG increases significantly when fructose is ingested simultaneously with fat compared to that observed following fat intake only ${ }^{6,7)}$. Therefore, simultaneous ingestion of fructose and fat may possibly exacerbate postprandial lipidemia. The levels of triglyceride-rich lipoproteins (TRL), namely chylomicrons (CM), very-low-density lipoproteins (VLDL) and their remnants, increase in the postprandial state, and postprandial hyperlipidemia is known to be a major risk factor for coronary heart disease, as proposed by Zilversmit ${ }^{8}$.

However, the influence of fructose intake on carbohydrate and lipid metabolism remains to be clarified, and very little information about the effects of fructose consumption on Japanese subjects is available. The World Health Organization has proposed that added sugars should provide no more than $10 \%$ of dietary energy ${ }^{9}$. At present, there are no evidencebased data regarding the upper limits of the intake of fructose, HFCS and sucrose for maintaining health in Japanese individuals ${ }^{10)}$. The consumers with the highest intake of fructose in the USA are adolescents and young adults ${ }^{11}$, which is most likely also the case in Japan. Therefore, we enrolled young healthy Japanese women as subjects and examined the acute effects of fructose consumption on postprandial lipidemia.

\section{Aim}

To elucidate the effects of simultaneous ingestion of fructose and fat on postprandial lipoprotein metabolism in young healthy Japanese women.

\section{Methods}

\section{Subjects}

Nine young healthy Japanese women with a normal weight, a normal ovarian cycle and an apoE phenotype $3 / 3$ phenotype were enrolled as participants. A normal weight was defined as a body mass index (BMI) of $\geq 18.5 \mathrm{~kg} / \mathrm{m}^{2}$ and $<25 \mathrm{~kg} / \mathrm{m}^{2}$. All of the subjects were nonsmokers, had no apparent acute or chronic illnesses and were not taking any medications or dietary supplements. This study was approved by the Institutional Review Board of the Sugiyama Jogakuen University School of Life Studies, and each subject provided their written informed consent for study participation.

\section{Anthropometric and Body Composition Measurement} Body weight and height were measured according to standard methods. Waist circumference was assessed as the abdominal girth at the level of the umbilicus, and the hip circumference was measured at the level of the greater trochanters. The waist-to-hip $(\mathrm{W} / \mathrm{H})$ ratio was calculated. Body composition, including the visceral fat area (VFA), was analyzed using an eight-polar bioelectrical impedance method with the InBody720 (BioSpace, Tokyo, Japan).

\section{Fructose, Glucose and the Fat Load Test}

Each subject was studied on four occasions. At each test trial, the subjects ingested one of four beverages containing either fructose or glucose $(0.5 \mathrm{~g} / \mathrm{kg}$ body weight each) with or without oral fat tolerance test (OFTT) cream (Jomo, Takasaki, Japan; $1 \mathrm{~g} / \mathrm{kg}$, $0.35 \mathrm{~g} / \mathrm{kg}$ as fat) in a randomized crossover design. OFTT cream was used as previously described ${ }^{12-14)}$. The four beverages were prepared as follows: Fru: 0.5 $\mathrm{g} / \mathrm{kg}$ of fructose (Nisshin Seito, Tokyo, Japan) mixed with no-sugar soda (Suntory, Tokyo, Japan); Fat: $1 \mathrm{~g} /$ $\mathrm{kg}$ of OFTT cream; Fru+Fat: fructose $(0.5 \mathrm{~g} / \mathrm{kg})$ mixed with no-sugar soda supplemented with OFTT cream $(1 \mathrm{~g} / \mathrm{kg})$; Glu: $0.5 \mathrm{~g} / \mathrm{kg}$ of glucose $(1.5 \mathrm{~mL} / \mathrm{kg}$ of Trelan-G75 ${ }^{\mathrm{TM}}, 1 \mathrm{~g} / 3 \mathrm{~mL}$ as glucose, Ajinomoto, Tokyo, Japan). All beverages were used at a concentration of $10 \%(\mathrm{w} / \mathrm{v})$.

\section{Experimental Design}

One of the four beverages mentioned above was administered after a 12-hour overnight fast. The subjects abstained from consuming caffeine or alcohol on the day before the experiment. Venous blood samples were obtained before (0 hours) and 0.5, 1, 2, 4 and 6 hours after ingestion. During the test, the subjects avoided exercise and eating, but had free access to water after one hour. All blood samples were obtained in the supine position. The experiments were conducted at least four weeks apart. There was an interval of four weeks between the test days to minimize the confounding effects of the subjects' menstrual status on lipid metabolism.

\section{Biochemical Analysis}

The serum samples were immediately refrigerated $\left(4^{\circ} \mathrm{C}\right)$ or frozen $\left(-80^{\circ} \mathrm{C}\right)$ until the analysis. Fructose was measured enzymatically (BioAssay Systems, CA, USA). Glucose was measured using a mutarotase GOD method (Wako, Osaka, Japan). Insulin was measured using a chemiluminescent enzyme immunoassay (Fujirebio, Tokyo, Japan). Insulin resistance was evaluated according to the homeostasis model assessment for insulin resistance (HOMA-IR) ${ }^{15)}$. The hemoglobin A1c (HbA1c) level was measured using a latex agglutination method (Fujirebio) and expressed 
as the National Glycohemoglobin Standardization Program (NGSP) value. The levels of free fatty acids (FFA) (Eiken Chemical, Tokyo, Japan) and lactate (Kyowa Medex, Tokyo, Japan) were measured enzymatically.

The level of total cholesterol (TC) was measured enzymatically (Sysmex, Hyogo, Japan). The level of high-density lipoprotein cholesterol (HDL-C) was measured using a direct method (Fujirebio). The level of low-density lipoprotein cholesterol (LDL-C) was calculated using the Friedewald formula. The level of small, dense LDL-C (sdLDL-C) was measured enzymatically (Denka Seiken, Tokyo, Japan). The TG level was measured enzymatically (Sekisui Medical, Tokyo, Japan). The remnant-like particle TG (RLP-TG) level was measured using an immunosorbent assay (Otsuka Pharmaceutical, Tokyo, Japan). The remnant lipoprotein cholesterol (RemL-C) level was measured using a homogenous assay (MetaboRead ${ }^{\mathrm{TM}}$, Kyowa Medex). In our previous studies, the serum concentration of RLP-C was measured using the immunoaffinity separation method ${ }^{16)}$; however, the $\mathrm{RLP}-\mathrm{C}$ concentration measured using this method is sometimes less than the lower limit of detection in young Japanese women ${ }^{14)}$. In the present study, the serum concentration of RemL-C was therefore measured using a newly developed homogenous assay ${ }^{16)}$, and no samples were found to contain a concentration below the detection limit. The lipoprotein(a) (Lp(a)) level was measured using a latex agglutination method (Sekisui Medical). The levels of apolipoproteins (apos) AI, AII, CII, CIII and $\mathrm{E}$ were measured using an immunoturbidimetric method (Sekisui Medical). The level of apoB48 was measured using a chemiluminescent enzyme immunoassay (Fujirebio). The apoE phenotype was measured using the isometric electrophoresis method (Phenotyping ApoE IEF System ${ }^{\text {TM }}$, Joko, Tokyo, Japan).

\section{Quantification of Postprandial Metabolism}

Postprandial metabolism was quantified by calculating the incremental area under the curve ( $\triangle \mathrm{AUC}$ ), which was defined as the difference between the area under the curve and the area below the baseline $(0$ hours) concentration from 0 to 6 hours, as previously described ${ }^{14)}$. Postprandial changes in the concentrations of TG, RLP-TG, RemL-C, apoB and apoB48 were calculated as the difference from the baseline mean value (as 0 at 0 hours) and shown as $\triangle \mathrm{TG}, \Delta$ RLP-TG, $\triangle$ RemL-C, $\triangle$ apoB and $\Delta$ apoB 48 , respectively.

\section{Statistics}

All data are expressed as the mean \pm SEM. The statistical analyses were performed using the SPSS ver. 19 software program (IBM, Tokyo, Japan). Differences in the time course compared with the fasting values were analyzed using the Friedman test followed by the Wilcoxon signed-rank test with the Bonferroni correction. The measured differences in the values at each time point in the four trials were assessed using the Kruskal-Wallis test followed by the Mann-Whitney U-test with the Bonferroni correction.

\section{Results}

The physical characteristics and fasting blood chemical data of the subjects are shown in Table $\mathbf{1}$. There were no significant differences in any of the physical characteristics between the four trials.

\section{Fructose, Glucose, Insulin, FFA and Lactate}

The serum concentrations of fasting and postprandial fructose, glucose, insulin, FFA and lactate in the four trials are presented in Table 2.

The serum fructose concentrations in the Fru and Fru+Fat trials were significantly increased at 0.5 , 1 and 2 hours compared with each fasting value $(p<$ 0.05). The concentration of fructose in the Fru trial was significantly higher than that observed in the Fru + Fat trial at 0.5 hours $(p<0.05)$. At 2 hours, the fructose concentration was still significantly higher in the Fru and Fru+Fat trials compared with that observed in the Glu and Fat trials ( $p<0.05$ each). The concentration of fructose in the Fru and Fru+Fat trials fell to the fasting level at 4 hours.

The serum concentration of glucose in the Glu trial was significantly higher than that observed in the other three trials at 0.5 hours $(p<0.05)$; however, it returned to the baseline value at 1 hour. Thereafter, there were no significant differences in the serum glucose concentration among the four trials.

The serum insulin concentration in the Glu trial was significantly higher than that observed in the other three trials at 0.5 hours $(p<0.05)$. However, at 2 hours, there were no significant differences in the serum insulin concentration among the four trials, and no differences from the baseline values were observed.

The serum concentration of FFA instantly decreased in the Glu, Fru and Fru+Fat trials, reaching a minimum at 1 hour. The FFA concentration in the Fru and Fru+Fat trials was higher than that observed in the Glu trial at 1 (Fru vs. Glu $p=0.19$, Fru+Fat vs. Glu $p=0.06$ ) and 2 hours (Fru vs. Glu $p=0.09$, Fru+ Fat vs. Glu $p<0.05)$. However, the FFA concentration in the Fat trial did not decrease after the fat load and 
Table 1. Anthropometric and clinical characteristics

\begin{tabular}{|c|c|c|c|c|}
\hline Trial & Fru & Fat & Fru+Fat & Glu \\
\hline Age (y) & $21.2 \pm 0.3$ & & & \\
\hline Height (cm) & $160.4 \pm 1.9$ & & & \\
\hline Weight (kg) & $52.8 \pm 2.3$ & $52.8 \pm 2.4$ & $52.7 \pm 2.3$ & $53.1 \pm 2.4$ \\
\hline BMI $\left(\mathrm{kg} / \mathrm{m}^{2}\right)$ & $20.5 \pm 0.7$ & $20.5 \pm 0.8$ & $20.5 \pm 0.7$ & $20.6 \pm 0.8$ \\
\hline Waist (cm) & $68.9 \pm 1.6$ & $70.2 \pm 1.7$ & $69.1 \pm 1.6$ & $71.1 \pm 1.6$ \\
\hline $\mathrm{W} / \mathrm{H}$ & $0.75 \pm 0.01$ & $0.78 \pm 0.02$ & $0.76 \pm 0.01$ & $0.77 \pm 0.01$ \\
\hline VFA $\left(\mathrm{cm}^{2}\right)$ & $24.5 \pm 4.3$ & $26.6 \pm 3.9$ & $26.3 \pm 3.8$ & $28.0 \pm 4.8$ \\
\hline HbA1c (NGSP) (\%) & $5.3 \pm 0.1$ & $5.3 \pm 0.1$ & $5.3 \pm 0.1$ & $5.4 \pm 0.1$ \\
\hline HOMA-IR & $1.5 \pm 0.2$ & $1.4 \pm 0.2$ & $1.5 \pm 0.2$ & $1.4 \pm 0.1$ \\
\hline $\mathrm{TC}(\mathrm{mg} / \mathrm{dL})$ & $176.6 \pm 6.9$ & $181.9 \pm 6.3$ & $182.3 \pm 9.0$ & $179.8 \pm 11.1$ \\
\hline LDL-C (mg/dL) & $99.2 \pm 7.3$ & $103.8 \pm 6.7$ & $104.6 \pm 8.4$ & $104.9 \pm 9.6$ \\
\hline sdLDL-C (mg/dL) & $23.3 \pm 1.8$ & $22.3 \pm 1.2$ & $25.1 \pm 2.4$ & $24.2 \pm 2.7$ \\
\hline HDL-C (mg/dL) & $63.6 \pm 3.7$ & $66.8 \pm 3.6$ & $64.6 \pm 3.2$ & $65.4 \pm 4.2$ \\
\hline $\mathrm{Lp}(\mathrm{a})(\mathrm{mg} / \mathrm{dL})$ & $7.6 \pm 6.3$ & & & \\
\hline apoAI (mg/dL) & $154.8 \pm 8.1$ & $154.9 \pm 7.0$ & $154.9 \pm 7.5$ & $153.7 \pm 10.8$ \\
\hline apoAII (mg/dL) & $27.1 \pm 1.3$ & $27.1 \pm 0.9$ & $28.2 \pm 1.3$ & $27.1 \pm 1.4$ \\
\hline apoCII (mg/dL) & $2.7 \pm 0.2$ & $2.5 \pm 0.2$ & $2.8 \pm 0.3$ & $2.3 \pm 0.2$ \\
\hline apoCIII (mg/dL) & $7.8 \pm 0.6$ & $7.7 \pm 0.5$ & $8.1 \pm 0.7$ & $7.3 \pm 0.7$ \\
\hline apoE (mg/dL) & $3.8 \pm 0.2$ & $4.1 \pm 0.3$ & $4.0 \pm 0.3$ & $3.8 \pm 0.3$ \\
\hline SBP (mmHg) & $99.1 \pm 1.8$ & $99.9 \pm 2.5$ & $97.7 \pm 2.3$ & $100.1 \pm 2.4$ \\
\hline DBP (mmHg) & $61.9 \pm 1.6$ & $64.8 \pm 2.2$ & $63.0 \pm 2.3$ & $64.1 \pm 3.1$ \\
\hline Pulse rate (beats/min) & $67.0 \pm 2.3$ & $68.0 \pm 2.4$ & $67.9 \pm 2.4$ & $65.6 \pm 2.1$ \\
\hline
\end{tabular}

All values are presented as the mean \pm SEM

was higher than that observed in the other three trials at 1 and 2 hours ( $p<0.05$ each).

The serum lactate concentration in the Fru and Fru+Fat trials significantly increased at 1 hour compared with each fasting value $(p<0.05)$. At 1 hour, the serum concentration of lactate in the Fru trial was higher than that observed in the Fat trial $(p<0.05)$ and the lactate concentration in the Fru+Fat trial was higher than that observed in the Glu and Fat trials ( $p$ $<0.05)$. However, there were no significant differences in the serum lactate concentration among the four trials thereafter.

\section{TG, RLP-TG and RemL-C}

The concentrations of fasting and postprandial TG, RLP-TG and RemL-C in the four trials are presented in Table 2 , and the time courses of $\Delta \mathrm{TG}, \Delta$ RLP-TG and $\triangle$ RemL-C are shown in Fig. 1A-C. $\Delta$ TG, $\Delta$ RLP-TG and $\Delta$ RemL-C were calculated because the baseline value of serum TG in the Glu trial was significantly lower than that in the Fru trial $(p<0.05)$. $\triangle \mathrm{TG}$ peaked at 2 hours in the Fat trial and at 4 hours in the Fru+Fat trial. The $\Delta \mathrm{TG}$ in the Fru+Fat trial was higher than that observed in the Fat trial at 4 and 6 hours $(p<0.05)$. The $\Delta \mathrm{TG}$ returned to the baseline value at 4 hours in the Fat trial and at 6 hours in the Fru+Fat trial. The $\triangle \mathrm{AUC}-\mathrm{TG}$ in the Fru+Fat trial was larger than that observed in the Fat trial $(p<0.05)$ (Table 3).

The $\triangle$ RLP-TG peaked at 2 hours in the Fat trial and at 4 hours in the Fru+Fat trial. The $\triangle$ RLP-TG in the Fru+Fat trial was higher than that observed in the Fat trial at 4 hours $(p<0.05)$. At 6 hours, the $\triangle$ RLPTG in the Fat trial, but not that in the Fru+Fat trial, returned to the baseline value. The $\triangle$ AUC-RLP-TG in the Fru+Fat trial had a tendency to be larger than that observed in the Fat trial $(p=0.15)$ (Table 3). The $\Delta$ RemL-C in the Fat trial peaked at 2 hours, then decreased and returned to the baseline value at 4 hours. In contrast, the $\triangle \mathrm{RemL}-\mathrm{C}$ in the Fru+Fat trial peaked at 4 hours and returned to the baseline value at 6 hours. The $\triangle$ RemL-C in the Fru+Fat trial was higher than that observed in the Fat trial at 4 hours ( $p$ $<0.05)$. The $\triangle$ AUC-RemL-C in the Fru+Fat trial tended to be larger than that observed in the Fat trial $(p=0.09)$ (Table 3).

\section{ApoB and ApoB48}

The concentrations of fasting and postprandial apoB and apoB48 in the four trials are presented in 
Table 2. Parameters before and after beverage ingestion

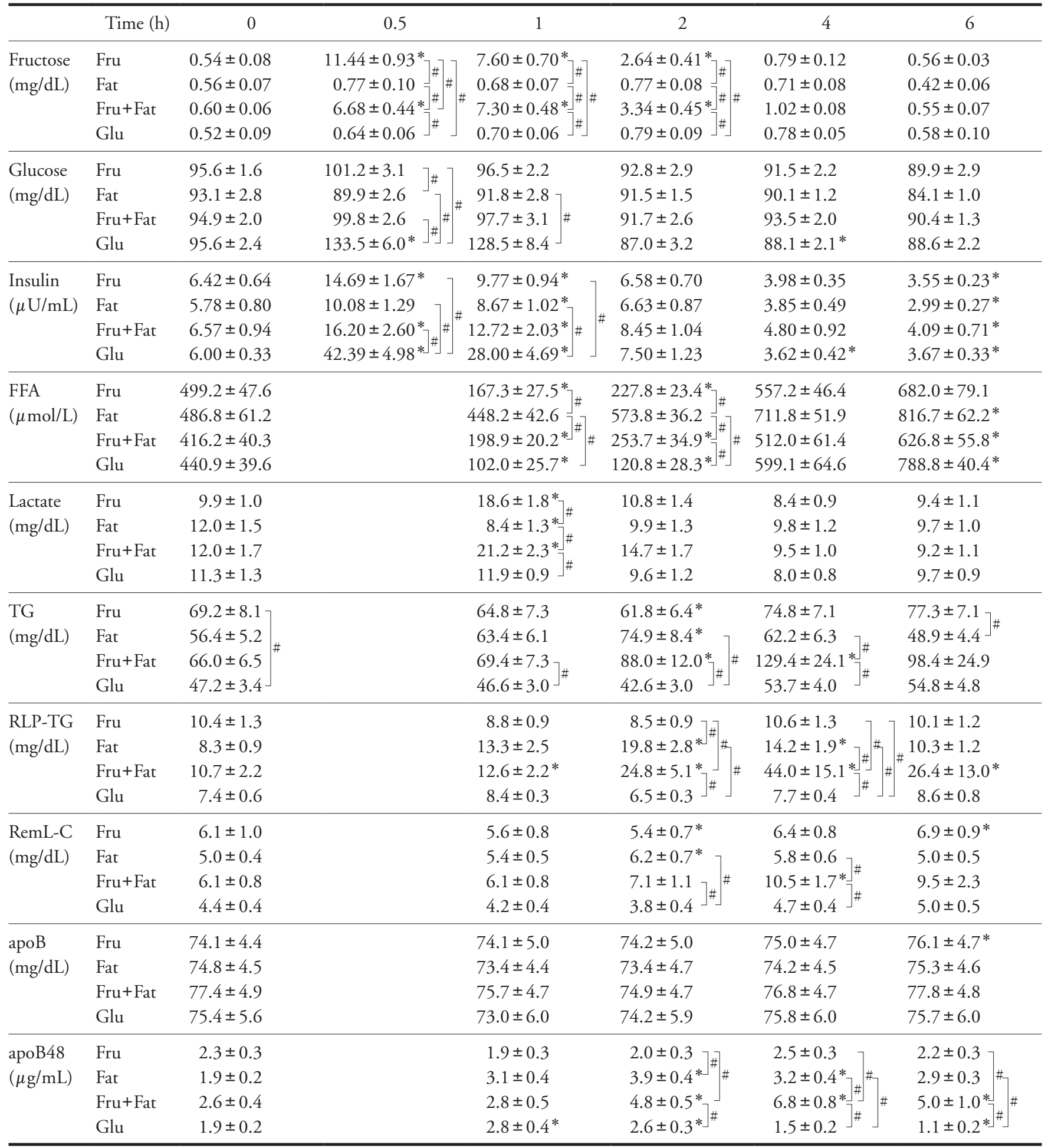

All values are presented as the mean \pm SEM. ${ }^{*} p<0.05$ compared to the fasting values. ${ }^{\#} p<0.05$ compared between the trials. 

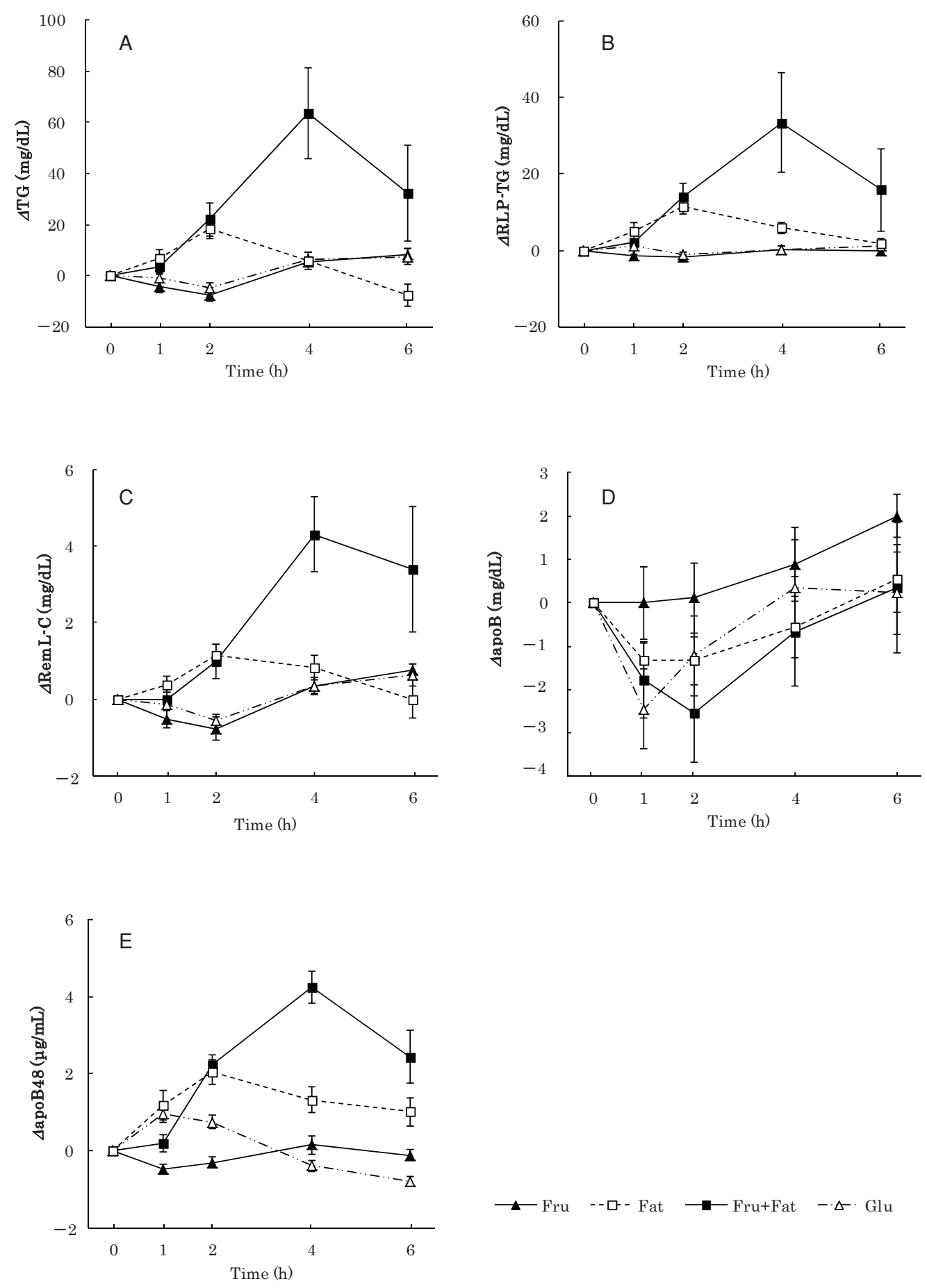

Fig.1. Postprandial $\Delta \mathrm{TG}(\mathrm{A}), \triangle \mathrm{RLP}-\mathrm{TG}(\mathrm{B}), \triangle \operatorname{RemL}-\mathrm{C}(\mathrm{C}), \triangle \mathrm{apoB}(\mathrm{D})$ and $\triangle \mathrm{apoB} 48$ (E). The values are presented as the mean \pm SEM.

Table 2, with the time courses of $\triangle \mathrm{apoB}$ and $\triangle \mathrm{apoB} 48$ shown in Fig. $1 D$ \& E. The $\triangle a p o B$ in the Fat, Fru+Fat and Glu trials was not significantly different than the fasting values observed over 6 hours. In contrast, the $\triangle \mathrm{apoB}$ in the Fru trial increased after 6 hours $(p<$
0.05). The $\triangle \mathrm{AUC}$-apoB did not differ among the four trials (Table 3).

The $\triangle \mathrm{apoB} 48$ in the Fat trial tended to increase at 1 hour $(p=0.11)$ and peaked at 2 hours. However, the $\triangle$ apoB48 in the Fru+Fat trial increased at 2 hours 
Table 3. The incremental areas under the curve ( $\triangle \mathrm{AUC}$ ) for TG, RLP-TG, RemL-C, apoB and apoB48

\begin{tabular}{|c|c|c|c|c|c|c|c|c|}
\hline Trial & $\begin{array}{l}\triangle \mathrm{AUC}-\mathrm{TG} \\
(\mathrm{mg} \cdot \mathrm{h} / \mathrm{dL})\end{array}$ & & $\begin{array}{c}\triangle \mathrm{AUC}-\mathrm{RLP}-\mathrm{TG} \\
(\mathrm{mg} \cdot \mathrm{h} / \mathrm{dL})\end{array}$ & & $\begin{array}{c}\triangle \text { AUC-RemL-C } \\
(\mathrm{mg} \cdot \mathrm{h} / \mathrm{dL})\end{array}$ & $\begin{array}{c}\triangle \mathrm{AUC} \text {-apoB } \\
(\mathrm{mg} \cdot \mathrm{h} / \mathrm{dL})\end{array}$ & \multicolumn{2}{|l|}{$\begin{array}{c}\triangle \mathrm{AUC} \text {-apoB48 } \\
(\mu \mathrm{g} \cdot \mathrm{h} / \mathrm{mL})\end{array}$} \\
\hline Fru & $3.6 \pm 11.2$ & \multirow{3}{*}{ * } & $-4.2 \pm 3.6$ & \multirow[b]{3}{*}{$*$} & $-0.2 \pm 1.1$ & $3.9 \pm 3.8$ & $-0.7 \pm 0.7\urcorner 7$ & \\
\hline Fat & $38.7 \pm 10.4$ & & $35.8 \pm 5.1$ & & $3.8 \pm 1.4 *$ & $-3.9 \pm 3.1$ & $7.9 \pm 1.3$ & * \\
\hline Fru+Fat & $195.8 \pm 62.9$ & & $105.6 \pm 40.7$ & & $13.5 \pm 4.1=$ & $-6.6 \pm 6.3$ & $14.4 \pm 1.4\rfloor$ & * \\
\hline
\end{tabular}

All values are presented as the mean \pm SEM. ${ }^{*} p<0.05$

and peaked at 4 hours $(p<0.05)$. The $\triangle$ apoB 48 in the Fru+Fat trial was significantly higher than that observed in the Fat trial at 4 and 6 hours $(p<0.05)$. The $\triangle \mathrm{apoB} 48$ in the Fat trial, but not that in the Fru + Fat trial, returned to the baseline value at 6 hours. The $\triangle \mathrm{AUC}$-apoB 48 in the Fru+Fat trial was significantly larger than that observed in the Fat trial $(p<$ 0.05) (Table 3). The $\triangle$ apoB48 in the Glu trial was slightly but significantly increased at 1 and 2 hours, then slightly decreased, then fell significantly below the baseline value at 6 hours. In contrast, the $\Delta$ apoB 48 in the Fru trial was not significantly different from the fasting value observed during the trial.

\section{Discussion}

The major finding of this study is that simultaneous ingestion of fructose and fat markedly enhanced postprandial lipidemia in young healthy Japanese women. In this study, the subjects ingested a moderate amount of fructose $(0.5 \mathrm{~g} / \mathrm{kg}$ body weight). Although other studies have employed $0.75-1.5 \mathrm{~g} / \mathrm{kg}$ of fructose $^{7,17)}$, these high amounts of fructose often induce malabsorption that causes gastrointestinal symptoms, for example bloating, abdominal pain and diarrhea ${ }^{18-20)}$. HFCS-sweetened beverages contain approximately $20-30 \mathrm{~g} / 500 \mathrm{~mL}$ of fructose ${ }^{21)}$. Therefore, we utilized a moderate amount of fructose $(0.5 \mathrm{~g} / \mathrm{kg})$ that could be ingested daily. The daily fat intake in Japanese adult women is approximately $50 \mathrm{~g}^{10)}$. Therefore, the amount of fat loaded in this study $(0.35 \mathrm{~g} / \mathrm{kg})$ corresponds to approximately $1 / 3$ of the daily fat consumption ${ }^{13)}$. We demonstrated in a previous study that the postprandial lipoprotein metabolism can be evaluated in subjects who consume this amount of fat ${ }^{12-14)}$. In daily dietary habits, fructose is usually consumed with fat. For example, some beverages provided by fast-food restaurants, such as milk shakes, contain fat and HFCS. Therefore, we studied the effects of simultaneous ingestion of fructose and fat on post- prandial lipoprotein and carbohydrate metabolism.

In the present study, the ingestion of fructose combined with fat led to a significantly higher rise and delayed peak in the serum TG, RLP-TG and RemL-C concentrations compared with the ingestion of fat only. Insulin increases the expression and activity of lipoprotein lipase (LPL), and subjects who consume fructose have lower levels of postprandial LPL activity than subjects who consume glucose ${ }^{22}$. The lower insulin excursion observed following fructose compared with glucose ingestion may result in a reduced activation of LPL, thus leading to a delayed TG clearance ${ }^{7)}$.

An interesting finding of this study is the change in the concentration of apoB 48 following the simultaneous ingestion of fructose and fat; the concentration of apoB48 significantly increased after 2 hours, and the peak was delayed compared with that observed following the ingestion of fat only, suggesting delayed intestinal absorption of dietary fat or secretion of CM from the intestine in addition to late clearance of intestinal TRL, namely CM and chylomicron-remnant (CM-R). The concentration of apoB48 observed following glucose intake was slightly but significantly increased at 1 hour, then declined steadily, falling below the baseline value at 6 hours, consistent with our previous results ${ }^{23)}$. In contrast, the concentration of apoB48 did not change following fructose intake during the trial. These results suggest that the ingestion of glucose, but not fructose, may transiently stimulate the synthesis and/or secretion of intestinal apoB48-containing lipoproteins.

Although postprandial dyslipidemia was previously regarded to be a consequence of delayed TRL clearance, emerging evidence implicates intestinal overproduction of apoB48-containing lipoproteins as a major contributor to postprandial dyslipidemia ${ }^{24)}$. In the present study, the concentration of apoB 48 markedly increased and its peak was delayed following intake of fructose combined with fat compared with 
that observed following intake of fat only. In addition, the concentration of apoB 48 was lower and its peak was earlier (at 2 hours) following intake of glucose combined with fat in our previous study ${ }^{23)}$ compared with that observed following intake of fructose combined with fat in the present study. These results suggest the retention of CM-R in the circulation following ingestion of fructose combined with fat compared with that observed following ingestion of glucose with fat. Delayed CM secretion from the intestine depends on differences in the absorption mechanisms of fructose and glucose. Fructose is transported into enterocytes through a specific fructose transporter, GLUT-5, located in the intestinal membrane. The absorption of fructose via facilitated diffusion, which does not require adenosine 5'-triphosphate, differs from that observed in the glucose absorption system ${ }^{25,26)}$. Therefore, the simultaneous ingestion of fructose and fat may have delayed the absorption of fat and the secretion of apoB48-containing TRL particles.

Fructose ingestion is known to acutely suppress plasma FFA and whole-body lipid oxidation ${ }^{27}$. The integrated postprandial inhibition of plasma FFA is of comparable magnitude following the ingestion of equivalent amounts of glucose and fructose, as shown in the present study. The slight increase in plasma insulin elicited by fructose may be sufficient to explain the inhibition of adipose tissue lipolysis, although this increase is quite modest compared with that observed following glucose ingestion.

In addition, the concentration of lactate increased following the ingestion of fructose (but not glucose) or fructose combined with fat. The increased lactate production most likely occurred because the fructokinase activity increased, the rate-limiting step for glycolysis (phosphofructokinase) was bypassed and the pyruvate kinase activity was stimulated by the accumulation of fructose-1-phosphate ${ }^{28)}$. However, part of the fructose absorbed into enterocytes may be converted into lactate and released into the portal circulation ${ }^{1)}$. In vitro data indicate that lactate rather than triose phosphate is the primary lipogenic precursor stimulated following fructose administration and that activation of pyruvate dehydrogenase by a highfructose diet is a major regulatory step in this process $^{29)}$. Simultaneously, fructose inhibits hepatic lipid oxidation, thereby favoring fatty acid reesterification and VLDL-TG synthesis ${ }^{30)}$. Triose phosphate produced from fructose is subsequently converted into pyruvate and oxidized into $\mathrm{CO}_{2}$ and $\mathrm{H}_{2} \mathrm{O}$ in the tricarboxylic acid cycle. A portion of the triose phosphate produced is converted into lactate, accounting for the increase in plasma lactate concentrations observed following fructose ingestion in the present study ${ }^{31}$.

Fructose has the same chemical formula as glucose, namely $\mathrm{C}_{6} \mathrm{H}_{12} \mathrm{O}_{6}$; however, its metabolism differs markedly from that of glucose due to its almost complete hepatic extraction and rapid hepatic conversion into glucose, glycogen, lactate and fat. A chronically high fructose intake has been shown to cause dyslipidemia and impair hepatic insulin sensitivity ${ }^{1)}$. A meta-analysis concluded that a fructose intake of $>$ $50 \mathrm{~g} /$ day (i.e., close to the average daily intake in the USA) is associated with increased postprandial TG excursions, while a fructose intake of $>100 \mathrm{~g} /$ day is associated with increased fasting TG levels ${ }^{32)}$. Diets containing $>15 \%$ of energy derived from fructose, compared with glucose, are consistently associated with increases in both fasting and postprandial TG concentrations in humans ${ }^{33-36)}$. Chronic consumption of fructose increases the deposition of visceral adipose tissue, while the consumption of glucose results primarily in an increased deposition of subcutaneous adipose tissue ${ }^{22)}$. The chronic consumption of fructose is also associated with an increased concentration of small, dense LDL particles ${ }^{37}$. The adverse effects of fructose may be explained by its hepatic metabolism, which is independent from the energy status, leading to unregulated hepatic fructose uptake and increased lipogenesis ${ }^{22)}$. As shown in the present study and a study conducted by Chong et al. ${ }^{7}$, after the ingestion of fructose, the peak plasma fructose concentration remains at approximately $10 \mathrm{mg} / \mathrm{dL}$, thus indicating that first-pass hepatic extraction is much higher than that observed in glucose ingestion. In the liver, fructose is metabolized into glyceraldehyde and dihydroxyacetone phosphate. The ability of fructose to bypass the primary regulatory step of glycolysis, the conversion of glucose-6-phosphate to fructose 1,6-bisphosphate controlled by phosphofructokinase, is of key importance. Therefore, while the glucose metabolism is negatively regulated by phosphofructokinase, fructose can continuously enter the glycolytic pathway in an unregulated manner ${ }^{38)}$. In the present study, the concentration of apoB(100) was increased in the trial of ingestion of fructose only, and not in the other three trials, thus suggesting that the ingestion of fructose may increase VLDL-apoB100 production in the liver.

Sex differences in fasting plasma TG concentrations are generally attributed to lower VLDL-TG concentrations in women. Increased clearance of VLDLTG in plasma is responsible for the lower VLDL-TG concentration, whereas a deceased VLDL-apoB100 secretion rate, combined with a shorter residence time 
of VLDL-apoB100 in plasma, is responsible for the lower VLDL-apoB100 concentrations observed in women $^{39)}$. In women, the liver secretes VLDL particles that contain more TG, and are therefore larger, than the VLDL particles secreted by the liver in men. The results of in vivo studies demonstrate faster removal of TG from large, TG-rich VLDL particles than from small, TG-poor particles ${ }^{40)}$. The present study demonstrated that the simultaneous ingestion of a moderate amount of fructose and fat, which can be ingested daily, markedly exacerbates postprandial exogenous lipidemia, even in young healthy women. Although postprandial lipidemia is a known risk factor for coronary heart disease ${ }^{8)}$, the influence of high fructose intake on health in young women has not been clarified. We are apprehensive of the deleterious effects of fructose ingestion on the health of this population. However, due to the relatively small number of subjects enrolled, the present results should be interpreted with caution.

\section{Conclusion}

Our present results demonstrated that the simultaneous ingestion of a moderate amount of fructose and fat markedly exacerbates postprandial exogenous lipidemia in young healthy Japanese women, thus suggesting that even a relatively modest amount of fructose intake may be excessive.

\section{Acknowledgements}

This work was supported by Grants-in-Aid for Scientific Research (Basic Research B, 2011 and Basic Research C, 2012) from the Ministry of Education, Culture, Sports, Science and Technology of Japan. We would like to thank Mr. Tatsuhiko Furuhashi for his technical assistance. We also give our thanks to Ms. Sonomi Ito, Ms. Yukie Ito, Ms. Kazue Osawa and Ms. Yuri Kato for their valuable technical cooperation.

\section{Conflicts of interest}

None.

\section{References}

1) Tappy L, Le KA: Metabolic effects of fructose and the worldwide increase in obesity. Physiol Rev, 2010; 90: 23-46

2) Ministry of Agriculture, Forestry and Fisheries, Japan, 2012. http://www.maff.go.jp/j/seisan/tokusan/kansho/ $\mathrm{pdf} / 23 \mathrm{sy}-3 . \mathrm{pdf}$ (in Japanese)

3) Kanai M: High fructose corn syrup's influence on sugar consumption in the Japanese diet. Journal of Rural Economics Back issues, 2000; 72 (Suppl): 146-150 (in Japa- nese)

4) Malik VS, Schulze MB, Hu FB: Intake of sugar-sweetened beverages and weight gain: a systematic review. Am J Clin Nutr, 2006; 84: 274-288

5) Stanhope KL, Griffen SC, Bair BR, Swarbrick MM, Keim NL, Havel PJ: Twenty-four-hour endocrine and metabolic profiles following consumption of high-fructose corn syrup, sucrose, fructose, and glucose sweetened beverages with meals. Am J Clin Nutr, 2008; 87: 1194-1203

6) Cohen JC, Schall R: Reassessing the effects of simple carbohydrates on the serum triglyceride responses to fat meals. Am J Clin Nutr, 1988; 48: 1031-1034

7) Chong MF, Fielding BA, Frayn KN: Mechanisms for the acute effect of fructose on postprandial lipemia. Am J Clin Nutr, 2007; 85: 1511-1520

8) ZilversmitDB: Atherogenesis: a postprandial phenomenon. Circulation, 1979; 60: 473-485

9) World Health Organization. Diet, nutrition and prevention of chronic diseases: report of a joint WHO/FAO Expert Consultation. World Health Organization, Geneva, 2003; 54-71

10) National Health and Nutrition Survey, Ministry of Health, Labour and Welfare, Japan, 2010. http://www. mhlw.go.jp/bunya/kenkou/sessyu-kijun.html (in Japanese)

11) Park YK, Yetley EA: Intakes and food sources of fructose in the United States. Am J Clin Nutr, 1993; 58 (Suppl): 737S-747S

12) Nabeno-Kaeriyama Y, Fukuchi Y, Hayashi S, Kimura T, Tanaka A, Naito M: Delayed postprandial metabolism of triglyceride-rich lipoproteins in obese young men compared to lean young men. Clin Chim Acta, 2010; 411: 1694-1699

13) Nabeno Y, Fukuchi $Y$, Matsutani $Y$, Naito M: Influence of aging and menopause on postprandial lipoprotein responses in healthy adult women. J Atheroscler Thromb, 2007; 14: $142-150$

14) Hashimoto S, Ootani K, Hayashi S, Naito M: Acute effects of shortly pre-versus postprandial aerobic exercise on postprandial lipoprotein metabolism in healthy but sedentary young women. J Atheroscler Thromb, 2011; 18: 891-900

15) Matthews DR, Hosker JP, Rudenski AS, Naylor BA, Treacher DF, Turner RC: Homeostasis model assessment: insulin resistance and beta-cell function from fasting plasma glucose and insulin concentrations in man. Diabetologia, 1985; 28: 412-419

16) Yoshida H, Kurosawa H, Hirowatari Y, Ogura Y, Ikewaki K, Abe I, Saikawa S, Domitsu K, Ito K, Yanai H, Tada N. Characteristic comparison of triglyceride-rich remnant lipoprotein measurement between a new homogenous assay (RemL-C) and a conventional immunoseparation method (RLP-C). Lipids Health Dis, 2008; 7: 18

17) Lê KA, Faeh D, Stettler R, Ith M, Kreis R, Vermathen P, Boesch C, Ravussin E, Tappy L: A 4-wk high-fructose diet alters lipid metabolism without affecting insulin sensitivity or ectopic lipids in healthy humans. Am J Clin Nutr, 2006; 84: 1374-1379

18) Rao SS, Attaluri A, Anderson L, Stumbo P: The Ability of the normal human small intestine to absorb fructose: evaluation by breath testing. Clin Gastroenterol Hepatol, 
2007; 5: 959-963

19) Skoog SM, Bharucha AE, Zinsmeister AR: Comparison of breath testing with fructose and high fructose corn syrups in health and IBS. Neurogastroenterol Motil, 2008; 20: 505-511

20) Gaby AR: Adverse effects of dietary fructose. Altern Med Rev, 2005; 10: 294-306

21) Latulippe ME, Skoog SM: Fructose malabsorption and intolerance: effects of fructose with and without simultaneous glucose ingestion. Crit Rev Food Sci Nutr, 2011; 51: 583-592

22) Stanhope KL, Schwarz JM, Keim NL, Griffen SC, Bremer AA, Graham JL, Hatcher B, Cox CL, Dyachenko A, Zhang W, McGahan JP, Seibert A, Krauss RM, Chiu S, Schaefer EJ, Ai M, Otokozawa S, Nakajima K, Nakano T, Beysen C, Hellerstein MK, Berglund L, Havel PJ: Consuming fructose-sweetened, not glucose-sweetened, beverages increases visceral adiposity and lipids and decreases insulin sensitivity in overweight/obese humans. J Clin Invest, 2009; 119: 1322-1334

23) Hashimoto S, Hayashi S, Yoshida A, Naito M: Acute effects of postprandial aerobic exercise on glucose and lipoprotein metabolism. J Atheroscler Thromb, 2013; 20: 204-213

24) Hsieh J, Hayashi AA, Webb J, Adeli K: Postprandial dyslipidemia in insulin resistance: mechanisms and role of intestinal insulin sensitivity. Atheroscler Suppl, 2008; 9: 7-13

25) Corpe CP, Burant CF, Hoekstra JH: Intestinal fructose absorption: clinical and molecular aspects. J Pediatr Gastroenterol Nutr, 1999; 28: 364-374

26) Douard V, Ferraris RP: Regulation of the fructose transporter GLUT5 in health and disease. Am J Physiol Endocrinol Metab, 2008; 295: E227-E237

27) Tappy L, Randin JP, Felber JP, Chiolero R, Simonson DC, Jequier E, DeFronzo RA: Comparison of thermogenic effect of fructose and glucose in normal humans. Am J Physiol, 260 (6 Pt 1): E718-E724

28) Hallfrisch J: Metabolic effects of dietary fructose. FASEB J, 1990; 4: 2652-2660

29) Park OJ, Cesar D, Faix D, Wu K, Shackleton CH, Hellerstein MK: Mechanisms of fructose-induced hypertriglyceridemia in the rat. Activation of hepatic pyruvate dehydrogenase through inhibition of pyruvate dehydrogenase kinase. Biochem J, 1992; 282: 753-757

30) Topping DL, Mayes PA: The immediate effects of insulin and fructose on the metabolism of the perfused liver. Changes in lipoprotein secretion, fatty acid oxidation and esterification, and lipogenesis and carbohydrate metabolism. Biochem J, 1972; 126: 295-311

31) Bjorkman O, Gunnarsson R, Hagström E, Felig P, Wahren $\mathrm{J}$ : Splanchnic and renal exchange of infused fructose in insulin-deficient type I diabetic patients and healthy controls. J Clin Invest, 1989; 83: 52-59

32) Livesey G, Taylor R: Fructose consumption and consequences for glycation, plasma triacylglycerol, and body weight: meta-analyses and meta-regression models of intervention studies. Am J Clin Nutr, 2008; 88: 1419-1437

33) Macdonald I: Influence of fructose and glucose on serum lipid levels in men and pre-and postmenopausal women. Am J Clin Nutr, 1966; 18: 369-372

34) Bantle JP, Raatz SK, Thomas W, Georgopulos A: Effects of dietary fructose on plasma lipids in healthy subjects. Am J Clin Nutr, 2000; 72: 1128-1134

35) Reiser S, Powell AS, Scholfield DJ, Panda P, Ellwood KC, Canary JJ: Blood lipids, lipoproteins, apolipoproteins, and uric acid in men fed diets containing fructose or high-amylose cornstarch. Am J Clin Nutr, 1989; 49: 832839

36) Hallfrisch J, Reiser S, Prather ES: Blood lipid distribution of hyperinsulinemic men consuming three levels of fructose. Am J Clin Nutr, 1983; 37: 740-748

37) Aeberli I, Zimmermann MB, Molinari L, Lehmann R, I'Allemand D, Spinas GA, Berneis K: Fructose intake is predictor of LDL particle size in overweight school children. Am J Clin Nutr, 2007; 86: 1174-1178

38) Basciano H, Federico L, Adeli K: Fructose, insulin resistance, and metabolic dyslipidemia. Nutr Metab, 2005; 2: 5

39) Magkos F, Patterson BW, Mohammed BS, Klein S, Mittendorfer B: Women produce fewer but triglyceride-richer very low-density lipoproteins than men. J Clin Endocrinol Metab, 2007; 92: 1311-1318

40) Fisher RM,Coppack SW, Humphreys SM, Gibbons GF, Frayn KN: Human triacylglycerol-rich lipoprotein subfractions as substrates for lipoprotein lipase. Clin Chim Acta, 1995; 236: 7-17 